\title{
Amplitude modes in three-dimensional spin dimers away from quantum critical point
}

\author{
M. Zhu $\odot,{ }^{1}$ M. Matsumoto, ${ }^{2}$ M. B. Stone,${ }^{3}$ Z. L. Dun, ${ }^{4}$ H. D. Zhou, ${ }^{4}$ T. Hong,${ }^{3}$ T. Zou, ${ }^{1}$ S. D. Mahanti, ${ }^{1}$ and X. Ke ${ }^{1, *}$ \\ ${ }^{1}$ Department of Physics and Astronomy, Michigan State University, East Lansing, Michigan 48824, USA \\ ${ }^{2}$ Department of Physics, Shizuoka University, Shizuoka 422-8529, Japan \\ ${ }^{3}$ Quantum Condensed Matter Division, Oak Ridge National Laboratory, Oak Ridge, Tennessee 37831, USA \\ ${ }^{4}$ Department of Physics and Astronomy, University of Tennessee, Knoxville, Tennessee 37996, USA
}

(Received 18 October 2018; revised manuscript received 24 September 2019; published 19 November 2019)

\begin{abstract}
Magnetic excitations of long-range ordered localized spin systems are generally well described by the linear spin-wave theory which accounts for phase fluctuations of the order parameter. In contrast, quasiparticles associated with amplitude fluctuations are only observed in a few quantum antiferromagnets close to a quantum critical point (QCP), where the ordered moment is suppressed significantly from its fully saturated value. Via inelastic neutron scattering measurements and extended spin-wave calculations, we present the observation and explanation of amplitude modes in the magnetic excitation spectra of three-dimensional $S=3 / 2$ spin dimers $\mathrm{Cr}_{2} \mathrm{TeO}_{6}$ and $\mathrm{Cr}_{2} \mathrm{WO}_{6}$, which nevertheless are away from the QCP with the ordered moment reduced by only $\sim 24 \%$. We show that the large spin of $\mathrm{Cr}^{3+}$ and narrow bandwidth of the amplitude modes render their detection even in polycrystalline samples. This paper suggests that amplitude modes are not the privilege of ordered spin systems in the vicinity of a QCP, but may be common excitation modes that can survive even away from the QCP, paving a way to search for and understand the physics of amplitude fluctuations in antiferromagnets beyond the quantum spin limit $(S=1 / 2)$.
\end{abstract}

DOI: 10.1103/PhysRevResearch.1.033111

\section{INTRODUCTION}

Quasiparticles in condensed-matter systems can behave analogously to those investigated in nuclear and particle physics. In recent years significant efforts have been invested to search for condensed-matter analogs of Majorana fermions [1] and Higgs bosons [2]. The idea of a Higgs boson was proposed by Anderson [3] even earlier than the prediction of such bosons in particle physics [4,5]. In condensed-matter systems Higgs-like quasiparticles, sometimes referred to as Higgs modes although the analog may be partially complete, are collective excitations associated with amplitude fluctuations of the order parameter. They have been found in certain superconductors [6-9], collections of ultracold atoms [10], density-wave systems [11-13], and quantum antiferromagnets [14-22].

For localized spin systems, in general, excitations of a long-range ordered state can be well described by the linear spin-wave theory (LSW), which gives rise to transverse Nambu-Goldstone modes ( $T$ mode) arising from phase fluctuations of the order parameter. When ordered spin systems are close to a quantum critical point (QCP), longitudinal modes ( $L$ mode), also known as amplitude modes, have been predicted to emerge in the magnetic excitation spectra

\footnotetext{
*Corresponding author: ke@pa.msu.edu

Published by the American Physical Society under the terms of the Creative Commons Attribution 4.0 International license. Further distribution of this work must maintain attribution to the author(s) and the published article's title, journal citation, and DOI.
}

[23-27]. A prototypical example is an $S=1 / 2$ spin dimer system consisting of two nearest-neighbor spins coupled antiferromagnetically. If the intradimer exchange dominates, the system exhibits a singlet $\left(S_{\text {tot }}=0\right)$ ground state with a gapped triplet $\left(S_{\text {tot }}=1\right)$ first excited state [28]. As interdimer interactions become stronger, the system undergoes a phase transition from a quantum disordered state to a long-range magnetically ordered state, in which longitudinal fluctuations in the magnitude of the sublattice magnetization give rise to amplitude modes. However, the experimental realization and observation of amplitude modes in quantum spin systems is rare, particularly limited to quasi-one-dimensional $S=1$ spin chains $[23,29,30]$, two-dimensional coupled $S=1 / 2$ two-leg spin ladders [20], and $S=1 / 2$ spin dimers [15,16,18]. This is in part because it is rare to have spin systems with the ground state close to a QCP and excitations within an appropriate energy range accessible using spectroscopic probes, and in part because amplitude modes have a finite lifetime, decaying into a pair of transverse Goldstone modes making an experimental observation difficult [31-34].

In this paper, we present an unanticipated observation of amplitude modes in the magnetic excitation spectra of $\mathrm{Cr}_{2} \mathrm{TeO}_{6}$ and $\mathrm{Cr}_{2} \mathrm{WO}_{6}$, both of which are away from a QCP, via inelastic neutron scattering (INS) measurements. The measured amplitude modes and transverse Goldstone modes can be well described using the extended spin-wave (ESW) theory [35]. Although these two compounds are not close to a QCP, the large spin quantum number of $\mathrm{Cr}^{3+}(S=3 / 2)$ and the narrow bandwidth of amplitude modes make the detection of amplitude modes possible in polycrystalline samples. This work provides insights into the search for quasiparticles involving Higgs-like amplitude fluctuations in 


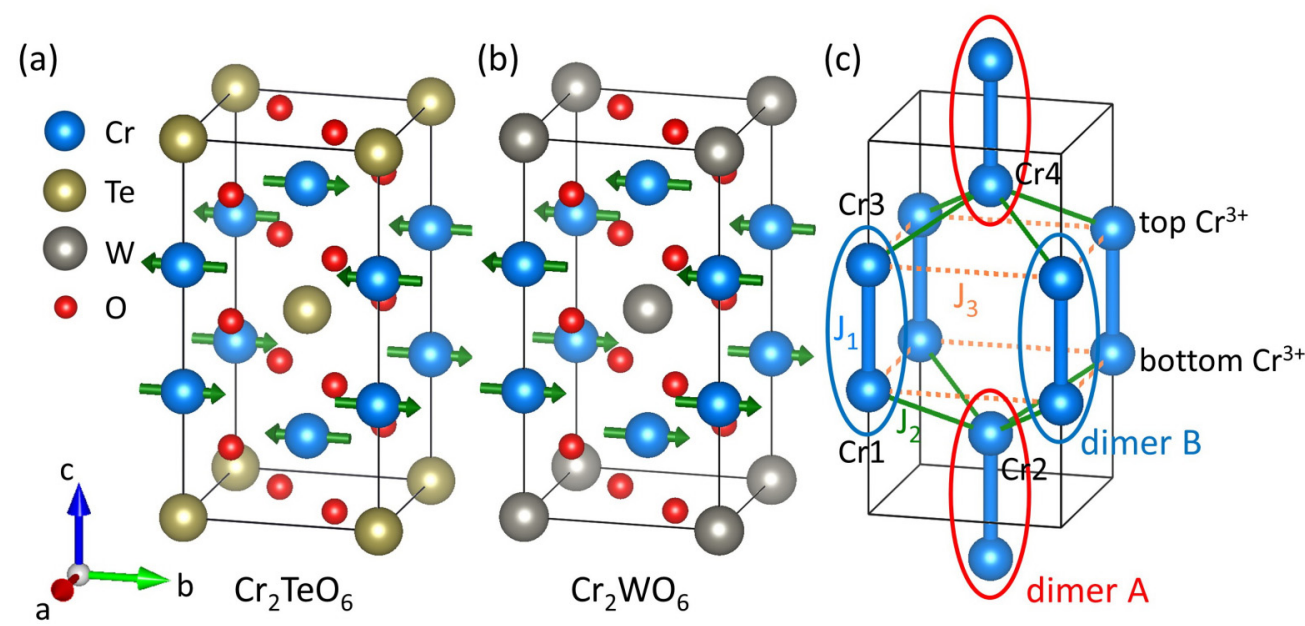

FIG. 1. Schematics of the crystal and magnetic structures of (a) $\mathrm{Cr}_{2} \mathrm{TeO}_{6}$ and (b) $\mathrm{Cr}_{2} \mathrm{WO}_{6}$. (c) Exchange interactions between $\mathrm{Cr}^{3+}$ ions. Two spins within a dimer are coupled by an antiferromagnetic exchange interaction $\left(J_{1}>0\right)$. The dimers interact with each other by interdimer interactions $\left(J_{2}\right.$ and $\left.J_{3}\right)$. The magnetic structure in the ordered phase indicates that $J_{3}<0$ (ferromagnetic) in both $\mathrm{Cr}_{2} \mathrm{WO}_{6}$ and $\mathrm{Cr}_{2} \mathrm{TeO}_{6}$, while $J_{2}$ is ferromagnetic $\left(J_{2}<0\right)$ in $\mathrm{Cr}_{2} \mathrm{WO}_{6}$ and antiferromagnetic $\left(J_{2}>0\right)$ in $\mathrm{Cr}_{2} \mathrm{TeO}_{6}$, respectively.

condensed-matter systems, particularly in spin dimers beyond $S=1 / 2$.

$\mathrm{Cr}_{2} \mathrm{TeO}_{6}$ and $\mathrm{Cr}_{2} \mathrm{WO}_{6}$ crystallize in an inverse trirutile structure composed of edge-sharing $\mathrm{CrO}_{6}$ octahedra separated by $\mathrm{TeO}_{6}$ or $\mathrm{WO}_{6}$ octahedra. As shown in Figs. 1(a) and 1(b), the nearest-neighbor $\mathrm{Cr}^{3+}$ ions with $S=3 / 2$ separated by $\sim 3 \AA$ are coupled antiferromagnetically $\left(J_{1}>0\right)$, which is much stronger than the further-neighbor interactions $(\sim 3.8 \AA)$ [36]. Thus, the two nearest-neighbor $\mathrm{Cr}^{3+}$ spins form $S=3 / 2$ dimers, whose dominant exchange interactions between $\mathrm{Cr}^{3+}$ spins are illustrated in Fig. 1(c). Both compounds exhibit a long-range antiferromagnetic order with Néel temperatures $T_{\mathrm{N}}=93 \mathrm{~K}$ for $\mathrm{Cr}_{2} \mathrm{TeO}_{6}$ and $T_{\mathrm{N}}=45 \mathrm{~K}$ for $\mathrm{Cr}_{2} \mathrm{WO}_{6}$, respectively, implying the important role of interdimer interactions. Interestingly, though the lattice parameters, bond lengths, and bond angles are very close, these two materials display very distinct ground-state magnetic structures [Figs. 1(a) and 1(b)], i.e., the interdimer interaction in $\mathrm{Cr}_{2} \mathrm{TeO}_{6}$ is antiferromagnetic (AF) $\left(J_{2}>0\right)$ whereas that in $\mathrm{Cr}_{2} \mathrm{WO}_{6}$ is ferromagnetic $\left(J_{2}<0\right)$ [37,38]. In spite of a highly insulating therefore ionic electronic ground state, the ordered moments of these two compounds are $\sim 2.3 \mu_{\mathrm{B}}$, which are reduced from the fully saturated value of $\mathrm{Cr}^{3+}\left(3 \mu_{\mathrm{B}}\right)$ [38] only by $\sim 24 \%$. This implies that they are not in the vicinity of a QCP, therefore the observation of amplitude modes is naively not anticipated.

\section{MATERIALS AND METHODS}

Polycrystalline samples of $\mathrm{Cr}_{2} \mathrm{TeO}_{6}$ and $\mathrm{Cr}_{2} \mathrm{WO}_{6}$ were synthesized using the solid-state chemistry reaction method reported earlier [37]. The quality of the samples has been verified by powder $\mathrm{x}$-ray and powder neutron diffraction measurements. The lattice parameters of $\mathrm{Cr}_{2} \mathrm{TeO}_{6}$ are $a=$ $b=4.545 \AA$ and $c=8.995 \AA$ and of $\mathrm{Cr}_{2} \mathrm{WO}_{6}$ are $a=b=$ $4.584 \AA$ and $c=8.853 \AA$, respectively. Inelastic neutron scattering (INS) measurements were performed using the SEQUOIA time-of-fight spectrometer at the Spallation Neu- tron Source at Oak Ridge National Laboratory. The incident neutron energy of most of the INS data presented in this paper was fixed as $E_{i}=65 \mathrm{meV}$ in order to cover a large enough area in energy and momentum $(E-|Q|)$ transfer space. The energy resolution is $\sim 1.9 \mathrm{meV}$ at elastic scattering [determined by the full width at half maximum (FWHM) of incoherent scattering]. Samples of $\sim 4 \mathrm{~g}$ each were loaded in a standard aluminum sample can with helium exchange gas and mounted onto a closed-cycle refrigerator. At each temperature, an identical empty sample can was measured for background subtraction.

\section{RESULTS}

\section{A. Magnetic excitation spectra at $T<T_{\mathrm{N}}$}

Figures 2(a) and 2(b) show the powder-averaged magnetic excitation spectra of polycrystalline $\mathrm{Cr}_{2} \mathrm{WO}_{6}$ and $\mathrm{Cr}_{2} \mathrm{TeO}_{6}$ as a function of energy transfer $E$ and momentum transfer $|Q|$ at $T=4 \mathrm{~K}$ measured by INS. In $\mathrm{Cr}_{2} \mathrm{WO}_{6}$, excitations have been observed at $0<E<10 \mathrm{meV}$ emanating from the magnetic Bragg reflection $(001)\left(|Q| \approx 0.72 \AA^{-1}\right)$ and equivalent $|Q|$ positions in the reciprocal space. No spin gap is observed within an energy resolution $\sim 0.92 \mathrm{meV}$ at elastic scattering measured using a lower incident energy $E_{i}=30 \mathrm{meV}$ (Fig. S1 [39]). The intensity of these excitations is strongest around $|Q| \approx 1.27 \AA^{-1}$ and $E \approx 9.0 \pm 0.4 \mathrm{meV}$. Strikingly, an additional gapped mode centered at $E \approx 12.0 \pm 0.4 \mathrm{meV}$ is also observed. Cuts through the data are presented in Figs. S2(a) and S2(b) of the Supplemental Material (SM) [39]. This mode is less dispersive [Fig. 2(a)], and the dependence of its intensity on $|Q|$ indicates that it is magnetic in origin [Fig. 3(a)]. Furthermore, there is another excitation mode around $E \approx 21.0 \pm 0.4 \mathrm{meV}$ as shown in Fig. 3(b). To better visualize these two additional modes, Fig. 4(a) shows cuts of the INS intensity as a function of $E$ with $|Q|$ integrated from 1.2 to $1.5 \AA^{-1}$. At $T=4 \mathrm{~K}$, three distinct modes are observed at $E \approx 9,12$, and $21 \mathrm{meV}$, respectively. 
(a)

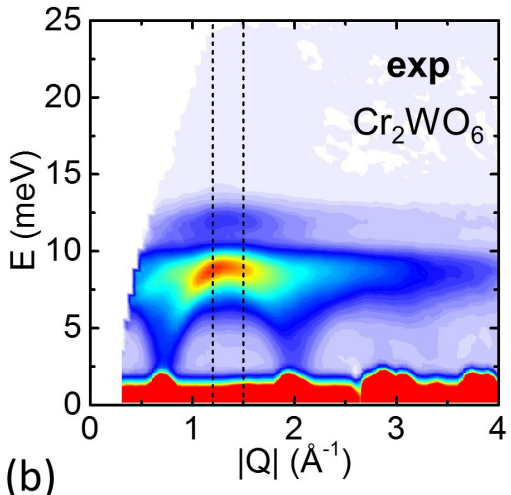

(b)

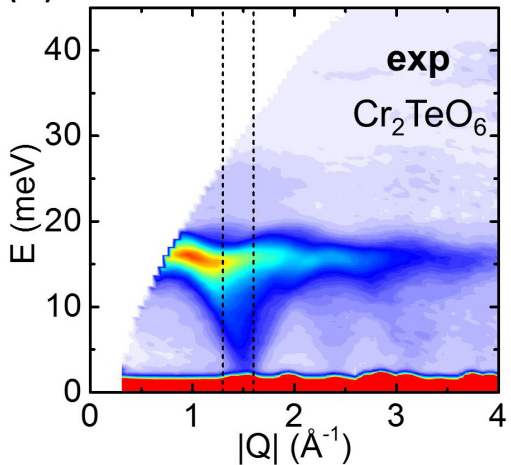

(c)

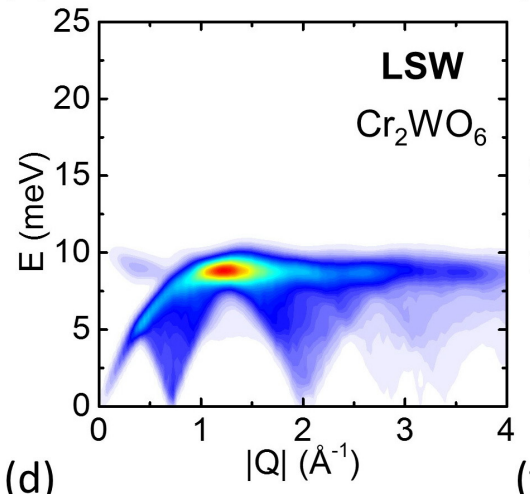

(d)

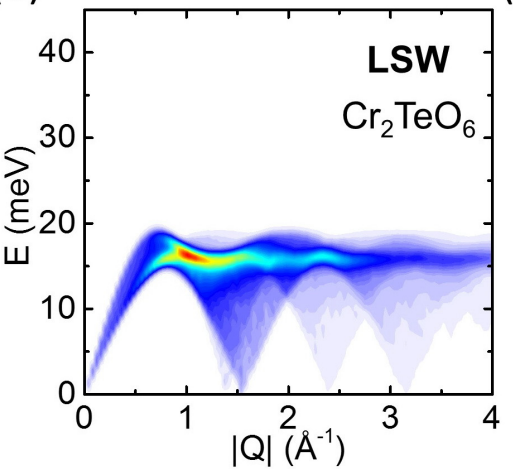

(e)

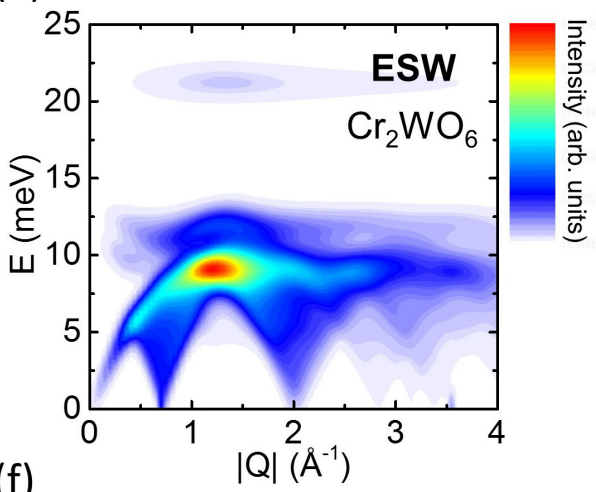

(f)

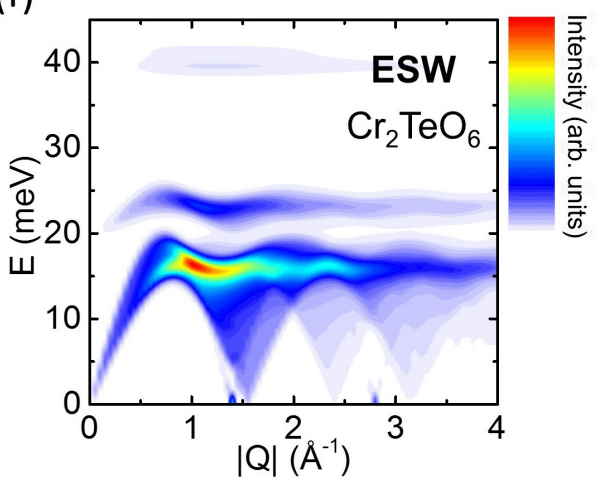

FIG. 2. (a), (b) Powder-averaged magnetic excitation spectra as a function of energy transfer $E$ and momentum transfer $|Q|$ of $\mathrm{Cr}_{2} \mathrm{TeO}_{6}$ and $\mathrm{Cr}_{2} \mathrm{WO}_{6}$ at $T=4 \mathrm{~K}$, respectively. Powder-averaged magnetic excitation spectra calculated by (c), (d) the LSW theory, and (e), (f) the ESW theory. Vertical dashed lines represent the $|Q|$ integration range in Fig. 4.

Similarly, in $\mathrm{Cr}_{2} \mathrm{TeO}_{6}$ there are modes in the magnetic excitation spectra but with a higher-energy scale $0<E<$ $19 \mathrm{meV}$ as seen in Fig. 2(b). In spite of the difference in the sign of $J_{2}$ in these two compounds, a gapped mode is also observed at $E \approx 23.0 \pm 1 \mathrm{meV}$ in $\mathrm{Cr}_{2} \mathrm{TeO}_{6}$ as shown in Figs. 3(c) and 4(b).

\section{B. Magnetic excitation spectra at $T>T_{\mathrm{N}}$}

We have also measured the powder-averaged magnetic excitations of $\mathrm{Cr}_{2} \mathrm{WO}_{6}$ and $\mathrm{Cr}_{2} \mathrm{TeO}_{6}$ at $T>T_{\mathrm{N}}$, as shown in Figs. 4 (red symbols) and 5. For $\mathrm{Cr}_{2} \mathrm{WO}_{6}$ measured at $T=60 \mathrm{~K}$, a broad peak is seen at low $E$ which might be ascribed to short-range correlations of the antiferromagnetic order persisting above $T_{\mathrm{N}}$ [Fig. 5(a)]. At temperatures (i.e., $T=160 \mathrm{~K}$ ) well above $T_{\mathrm{N}}$ (Fig. S3 [39]), short-range correlations of the antiferromagnetic order disappear. The triplet excitations of the dimers propagate via interdimer interactions and form gapped magnon modes. Similarly, a broad distribution of magnon intensity associated with short-range magnetic correlation is also observed in $\mathrm{Cr}_{2} \mathrm{TeO}_{6}$ when measured at $120 \mathrm{~K}$ [Fig. 5(b)], which is slightly above $T_{\mathrm{N}}$. In contrast, (a)

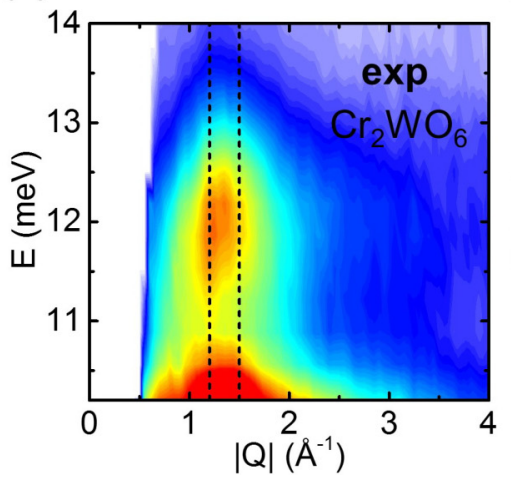

(b)

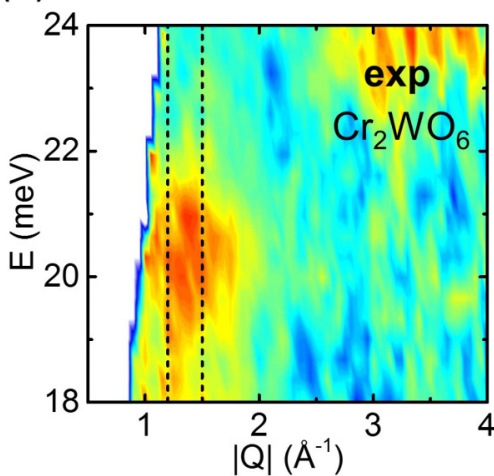

(c)

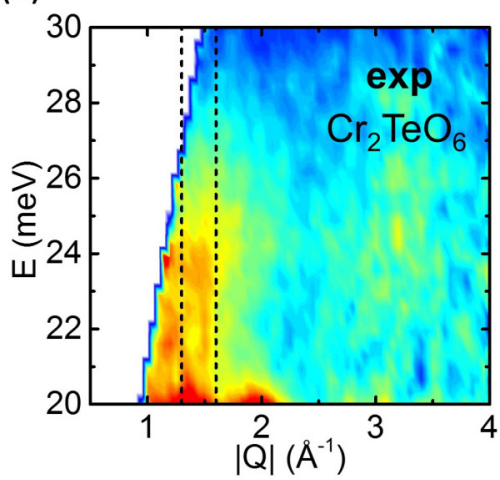

FIG. 3. Powdered-averaged magnetic excitation spectra measured by INS in Figs. 2(a) and 2(b) with a different color scale to highlight the modes at (a) $E \approx 12 \mathrm{meV}$ and (b) $E \approx 21 \mathrm{meV}$ in $\mathrm{Cr}_{2} \mathrm{WO}_{6}$ and (c) over 20-28 $\mathrm{meV}$ in $\mathrm{Cr}_{2} \mathrm{TeO}_{6}$. Vertical dashed lines represent the $|Q|$ integration range in Fig. 4. 
(a)

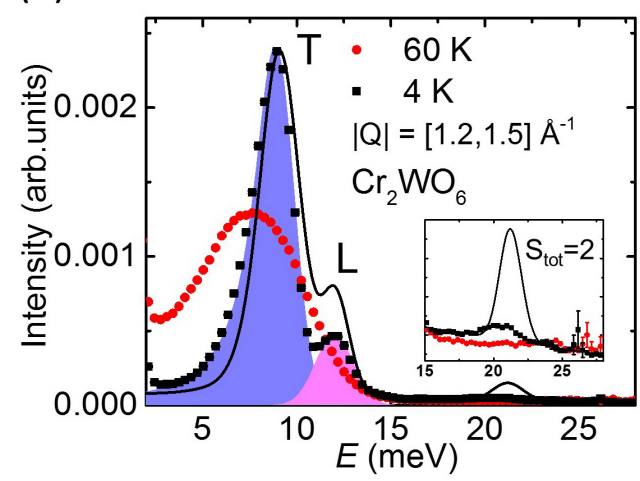

(b)

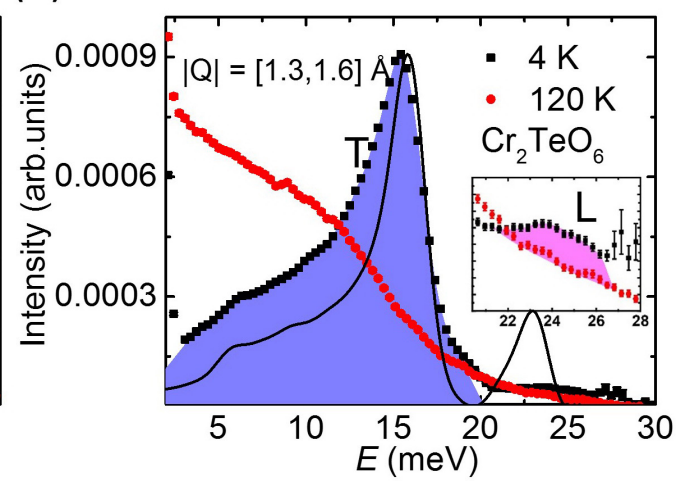

FIG. 4. INS intensity as a function of energy transfer $E$ at $T=4 \mathrm{~K}$ (black square) and $T>T_{\mathrm{N}}$ (red circle) of (a) $\mathrm{Cr}_{2} \mathrm{WO}_{6}$ and (b) $\mathrm{Cr}_{2} \mathrm{TeO}_{6}$. Insets display the zoom-in view of $15<E<28 \mathrm{meV}$ for $\mathrm{Cr}_{2} \mathrm{WO}_{6}$, and $20.5<E<28 \mathrm{meV}$ for $\mathrm{Cr}_{2} \mathrm{TeO}_{6}$. Solid curves show the INS intensity

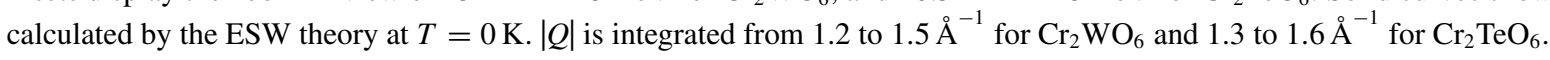

the higher-energy modes at $E \approx 12$ and $21 \mathrm{meV}$ observed in $\mathrm{Cr}_{2} \mathrm{WO}_{6}$ and at $E \approx 23 \mathrm{meV}$ in $\mathrm{Cr}_{2} \mathrm{TeO}_{6}$ at low temperature have been completely suppressed, affirming their magnetic character.

\section{DISCUSSIONS}

\section{A. Linear spin-wave theory without anisotropy}

To understand the magnetic excitations in $\mathrm{Cr}_{2} \mathrm{WO}_{6}$ and $\mathrm{Cr}_{2} \mathrm{TeO}_{6}$ measured by INS, first, we discuss the results calculated by the linear spin-wave theory (LSW). As shown in Fig. 1(c), there are four $\mathrm{Cr}$ atoms in the tetragonal unit cell. The Heisenberg Hamiltonian of the systems with a minimum of three exchange parameters $J_{1}, J_{2}$, and $J_{3}$ [39] has the form of

$$
\begin{aligned}
H=J_{1} \sum_{n} \sum_{i}[ & \left.S_{i n}^{(1) A} \cdot S_{i n}^{(3) B}+\frac{1}{2}\left\{S_{i n}^{(2) A} \cdot S_{i n-1}^{(4) B}+S_{i n}^{(4) B} \cdot S_{i n+1}^{(2) A}\right\}\right] \\
& +J_{2} \sum_{n} \sum_{\langle i, j\rangle}\left[S_{i n}^{(1) A} \cdot S_{j n}^{(2) A}+S_{i n}^{(3) B} \cdot S_{j n}^{(4) B}\right] \\
& +J_{3} \sum_{n} \sum_{\langle\langle i, j\rangle\rangle}\left[S_{i n}^{(1) A} \cdot S_{j n}^{(1) A}+S_{i n}^{(2) A} \cdot S_{j n}^{(2) A}\right. \\
& \left.+S_{i n}^{(3) B} \cdot S_{j n}^{(3) B}+S_{i n}^{(4) B} \cdot S_{j n}^{(4) B}\right]
\end{aligned}
$$

(a)

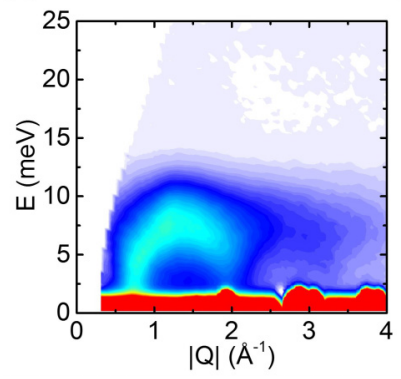

(b)

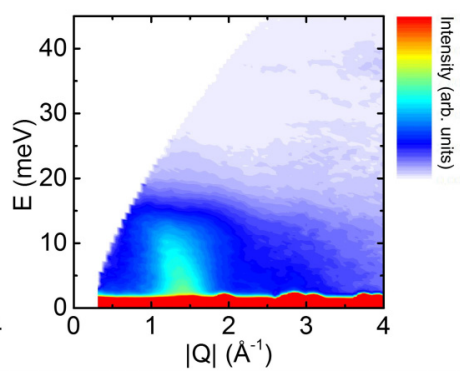

FIG. 5. Powder-averaged magnetic excitation spectra measured at $T>T_{\mathrm{N}}$ in (a) $\mathrm{Cr}_{2} \mathrm{WO}_{6}$ at $T=60 \mathrm{~K}$, and (b) $\mathrm{Cr}_{2} \mathrm{TeO}_{6}$ at $120 \mathrm{~K}$, respectively, with incident neutron energy $E_{i}=65 \mathrm{meV}$. for $\mathrm{Cr}_{2} \mathrm{WO}_{6}$ and

$$
\begin{aligned}
H=J_{1} \sum_{n} \sum_{i}[ & \left.S_{i n}^{(1) A} \cdot S_{i n}^{(3) B}+\frac{1}{2}\left\{S_{i n}^{(2) B} \cdot S_{i n-1}^{(4) A}+S_{i n}^{(4) A} \cdot S_{i n+1}^{(2) B}\right\}\right] \\
& +J_{2} \sum_{n} \sum_{\langle i, j\rangle}\left[S_{i n}^{(1) A} \cdot S_{j n}^{(2) B}+S_{i n}^{(3) B} \cdot S_{j n}^{(4) A}\right] \\
& +J_{3} \sum_{n} \sum_{\langle\langle i, j\rangle\rangle}\left[S_{i n}^{(1) A} \cdot S_{j n}^{(1) A}+S_{i n}^{(4) A} \cdot S_{j n}^{(4) A}\right. \\
& \left.+S_{i n}^{(2) B} \cdot S_{j n}^{(2) B}+S_{i n}^{(3) B} \cdot S_{j n}^{(3) B}\right]
\end{aligned}
$$

for $\mathrm{Cr}_{2} \mathrm{TeO}_{6}$, respectively. $n$ represents the $n$th unit cell along the $c$ direction. $\langle i, j\rangle$ implies that each bond is counted once. $A$ and $B$ are indices for sublattices $A$ and $B$. For example, $S_{\text {in }}^{(1) A}$ represents the spin of $\mathrm{Cr} 1$ at site $i$ in the $n$th unit cell. The powdered-averaged magnetic excitation spectra calculated using an isotropic Hamiltonian are shown in Figs. 2(c) and 2(d). $J_{1}, J_{2}$, and $J_{3}$ have been chosen to reproduce the dominant features (e.g., bandwidth, spectral weight maximum) in the powdered-averaged magnetic excitation spectra measured by INS (see Ref. [39] for more discussions).

The magnetic excitations below $10 \mathrm{meV}$ can be well explained by the LSW theory taking into account exchange interactions $J_{1}, J_{2}$, and $J_{3}$ defined in Fig. 1(c), where the powder average of two magnetic excitation branches accounts for the observed spectra [Figs. 2(c) and S5(a) [39]]. Thus, the magnetic excitations at $0<E<10 \mathrm{meV}$ are characteristic of the transverse Goldstone modes originating from spontaneously broken rotational symmetry in the magnetically ordered state. Similarly, in $\mathrm{Cr}_{2} \mathrm{TeO}_{6}$, the excitations in the energy range $0<E<19 \mathrm{meV}$ can also be described by the LSW theory [Figs. 2(d) and S5(b) [39]].

It is important to point out that the additional gapped modes at 12 and $21 \mathrm{meV}$ in $\mathrm{Cr}_{2} \mathrm{WO}_{6}$ and at $23 \mathrm{meV}$ in $\mathrm{Cr}_{2} \mathrm{TeO}_{6}$ cannot be captured by these LSW theory calculations with an isotropic Heisenberg Hamiltonian [Figs. 2(c) and 2(d)].

\section{B. Linear spin-wave theory with anisotropy}

Next, we consider the LSW theory with an anisotropy term in the spin Hamiltonian. We have performed LSW calculations for $\mathrm{Cr}_{2} \mathrm{WO}_{6}$ and $\mathrm{Cr}_{2} \mathrm{TeO}_{6}$ with a single-ion anisotropy 

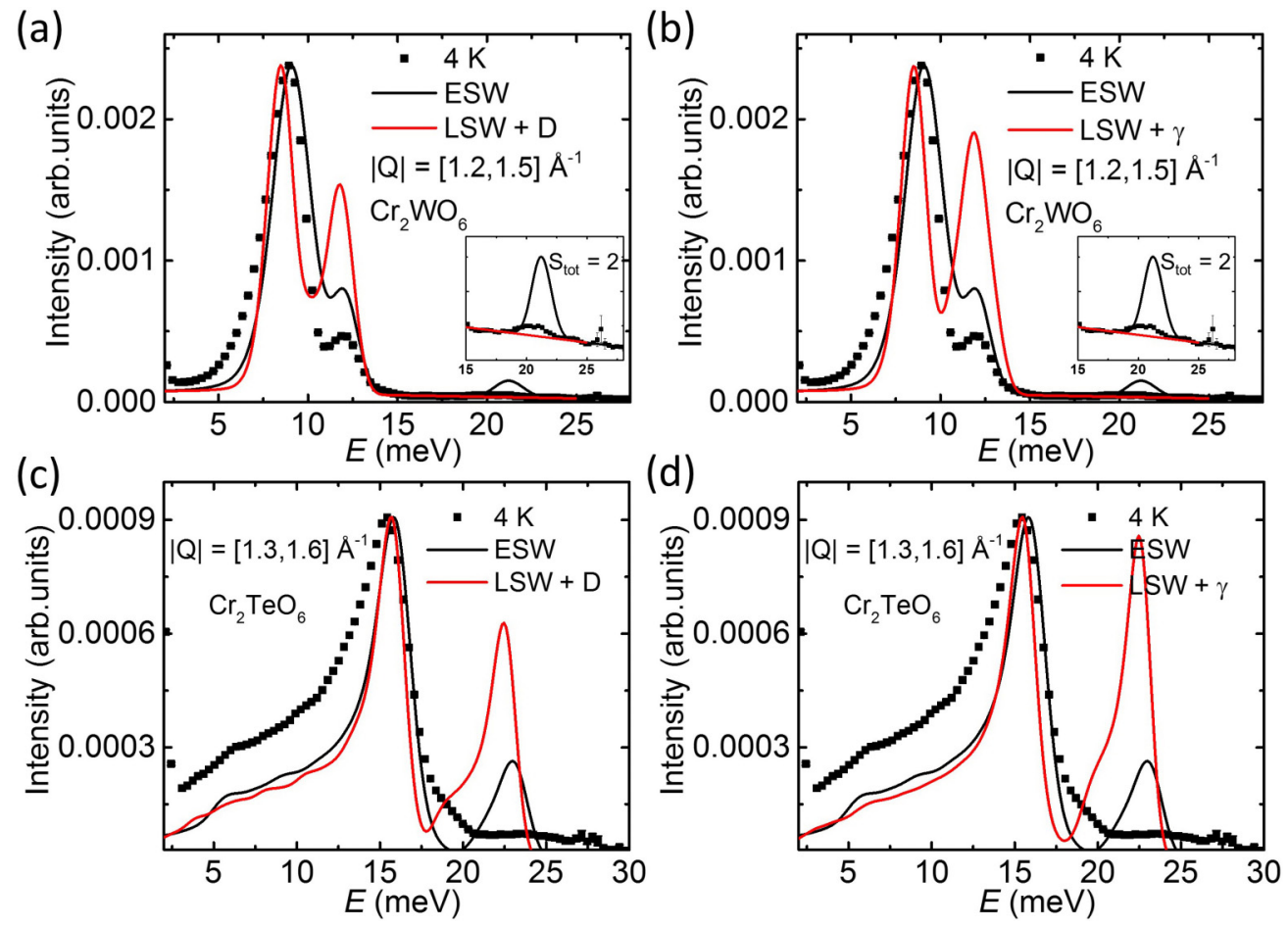

FIG. 6. INS intensity as a function of energy transfer $E$ of $\mathrm{Cr}_{2} \mathrm{WO}_{6}$ and $\mathrm{Cr}_{2} \mathrm{TeO}_{6}$ measured by INS (black symbols), $|Q|$ integrated from 1.2 to $1.5 \AA^{-1}$ in $\mathrm{Cr}_{2} \mathrm{WO}_{6}$ and from 1.3 to $1.6 \AA^{-1}$ in $\mathrm{Cr}_{2} \mathrm{TeO}_{6}$, respectively. The black curve is calculated by the ESW theory. The red curve is calculated by the LSW theory with an anisotropic term in the Hamiltonian: (a), (c) Single-ion anisotropy $D=1.2 \mathrm{meV}$ for $\mathrm{Cr}_{2} \mathrm{WO}_{6}$ and $D=2.5 \mathrm{meV}$ for $\mathrm{Cr}_{2} \mathrm{TeO}_{6}$, respectively; (b), (d) XXZ-type exchange anisotropy $\gamma=0.7$ for both compounds. Insets show the mode at $\sim 21$ $\mathrm{meV}$ that can be reproduced only by the ESW theory. A linear background has been added in (a) and (b).

$D$ and an XXZ-type exchange anisotropy $\gamma$ [defined in the spin Hamiltonian $J_{n}\left(S_{x}^{i} S_{x}^{j}+S_{y}^{i} S_{y}^{j}+\gamma S_{z}^{i} S_{z}^{j}\right)$, where $J_{n}$ represents the intra- $(n=1)$ and interdimer $(n=2,3)$ interactions], respectively. In both compounds, inclusion of an easy-plane type anisotropy $D$ or an exchange anisotropy $\gamma$ leads to a splitting of the transverse modes.

For $\mathrm{Cr}_{2} \mathrm{WO}_{6}$, the exchange parameters $J_{1}, J_{2}$, and $J_{3}$ and anisotropy constants $D$ or $\gamma$ have been finely tuned to capture (i) transverse modes with an intensity maximum around $9 \mathrm{meV}$, and (ii) gapped transverse modes with an intensity maximum around $12 \mathrm{meV}$. However, the calculated magnetic intensity around $12 \mathrm{meV}$ is much stronger than that observed in Fig. 2(a), as shown in Figs. 6(a) and 6(b) where $E$ cuts of the powdered-averaged magnetic spectra measured by INS, and the calculated results given by LSW theory with an anisotropy term and by ESW theory (to be discussed next) are plotted for a quantitative comparison. In addition, very importantly, the second gapped mode at $E \approx 21 \mathrm{meV}$ shown in Fig. 3(b) is absent in all LSW calculations. These two points are difficult to improve by adjusting the strength of exchange coupling or anisotropy.

The effect of anisotropy on the LSW results of $\mathrm{Cr}_{2} \mathrm{TeO}_{6}$ is qualitatively similar to $\mathrm{Cr}_{2} \mathrm{WO}_{6}$. The exchange parameters $J_{1}, J_{2}$, and $J_{3}$ and anisotropic coefficients $D$ or $\gamma$ have been finely tuned to capture (i) transverse modes with an intensity maximum at $\sim 16 \mathrm{meV}$, and (ii) gapped transverse modes at $\sim 23 \mathrm{meV}$. To compare the INS intensity quantitatively, $E$ cuts of the powdered-averaged magnetic spectra measured by INS, together with the calculated results given by the LSW theory with an anisotropy term involved and the ESW theory, are shown in Figs. 6(c) and 6(d). Though LSW theory can give rise to modes at $\sim 23 \mathrm{meV}$, the intensity is too strong compared with that observed experimentally.

Therefore, we conclude that the single-ion anisotropy or XXZ-type exchange anisotropy cannot account for all the experimental observations.

\section{Extended spin-wave theory}

Since the weakly dispersing high-energy gapped modes in the magnetic excitation spectra of $\mathrm{Cr}_{2} \mathrm{WO}_{6}$ and $\mathrm{Cr}_{2} \mathrm{TeO}_{6}$ cannot be fully explained within LSW approximations where only phase fluctuations of the order parameter have been involved, these gapped modes might potentially be attributed to amplitude modes associated with fluctuations of the magnitude of ordered moments. In order to understand the nature of these magnetic excitations, we have extended the linear spinwave calculations for interacting spin dimer systems [35]. The extended spin-wave (ESW) theory was originally introduced to study multipole dynamics of quadrupole ordered phases in $\mathrm{CeB}_{6}$ [35]. It is equivalent to the bond operator formulation introduced to study excited states in bilayer spin systems [40] and interacting spin dimers [27,41]. The present formulation has an advantage for investigating magnetic excitations in complicated systems such as $S=3 / 2$ spin dimers, where there are 16 local states.

Starting from the crystal structure of $\mathrm{Cr}_{2} \mathrm{WO}_{6}$ and $\mathrm{Cr}_{2} \mathrm{TeO}_{6}$ shown in Fig. 1(c), the Hamiltonian for interacting $S=3 / 2$ 
TABLE I. Exchange parameters and sublattice magnetic moment of $\mathrm{Cr}_{2} \mathrm{WO}_{6}$ and $\mathrm{Cr}_{2} \mathrm{TeO}_{6}$ obtained by ESW theory calculations (Sec. VD [39]). The ordered moment has been normalized to the full moment of $\mathrm{Cr}^{3+}\left(M_{s}=3 \mu_{\mathrm{B}}\right)$.

\begin{tabular}{lcrcc}
\hline \hline & $J_{1}(\mathrm{meV})$ & $J_{2}(\mathrm{meV})$ & $J_{3}(\mathrm{meV})$ & $M / M_{s}$ \\
\hline $\mathrm{Cr}_{2} \mathrm{WO}_{6}$ & $5.25(67)$ & $-0.475(20)$ & -0.10 & 0.794 \\
$\mathrm{Cr}_{2} \mathrm{TeO}_{6}$ & $9.40(54)$ & $1.035(46)$ & -0.10 & 0.820 \\
\hline \hline
\end{tabular}

spin dimers is given by the following general form,

$$
H=\sum_{i} \sum_{\mu} H_{\text {intra }}(i, \mu)+\sum_{\left\langle i, \mu, j, \mu^{\prime}\right\rangle} H_{\text {inter }}\left(i, \mu, j, \mu^{\prime}\right) .
$$

The first term $H_{\text {intra }}(i, \mu)$ represents a Hamiltonian of a dimer on the $\mu(=A, B)$ sublattice [Fig. 1(c)] in the $i$ th unit cell. It is written as

$$
H_{\text {intra }}(i, \mu)=J_{1} \boldsymbol{S}_{i \mu l} \cdot \boldsymbol{S}_{i \mu r} .
$$

$S_{i \mu \gamma}$ represents the $S=3 / 2$ spin operator at the $\gamma[=l, r$ top and bottom spins of the dimer in Fig. 1(c)] site in a dimer. The second term $H_{\text {inter }}\left(i, \mu, j, \mu^{\prime}\right)$ is the Hamiltonian for interdimer interactions between dimers on the $\mu$ and $\mu^{\prime}$ sublattices in the $i$ th and $j$ th unit cell, respectively. It is expressed as

$$
H_{\text {inter }}\left(i, \mu, j, \mu^{\prime}\right)=\sum_{\gamma, \gamma^{\prime}=l, r} J_{i \mu \gamma, j \mu^{\prime} \gamma^{\prime}} \boldsymbol{S}_{i \mu \gamma} \cdot \boldsymbol{S}_{j \mu^{\prime} \gamma^{\prime}},
$$

where $J_{i \mu \gamma, j \mu^{\prime} \gamma^{\prime}}$ is the exchange coupling constant. In Eq. (3), the summation $\sum_{\left\langle i \mu j \mu^{\prime}\right\rangle}$ is taken over pairs of spins interacting via $J_{2}$ and $J_{3}$.

First, we solve the Hamiltonian with a mean-field approximation on the basis of dimer states,

$$
\begin{aligned}
H_{A}= & J_{1} \boldsymbol{S}_{A l} \cdot \boldsymbol{S}_{A r}+4 J_{2} \boldsymbol{S}_{A l} \cdot\left\langle\boldsymbol{S}_{B r}\right\rangle+4 J_{2} \boldsymbol{S}_{A r} \cdot\left\langle\boldsymbol{S}_{B l}\right\rangle \\
& +4 J_{3} \boldsymbol{S}_{A l} \cdot\left\langle\boldsymbol{S}_{A l}\right\rangle+4 J_{3} \boldsymbol{S}_{A r} \cdot\left\langle\boldsymbol{S}_{A r}\right\rangle, \\
H_{B}= & J_{1} \boldsymbol{S}_{B l} \cdot \boldsymbol{S}_{B r}+4 J_{2} \boldsymbol{S}_{B l} \cdot\left\langle\boldsymbol{S}_{A r}\right\rangle+4 J_{2} \boldsymbol{S}_{B r} \cdot\left\langle\boldsymbol{S}_{A l}\right\rangle \\
& +4 J_{3} \boldsymbol{S}_{B l} \cdot\left\langle\boldsymbol{S}_{B l}\right\rangle+4 J_{3} \boldsymbol{S}_{B r} \cdot\left\langle\boldsymbol{S}_{B r}\right\rangle .
\end{aligned}
$$

Here, $\langle\cdots\rangle$ represents the expectation value of the spin operator obtained in the mean-field ground state. We diagonalize the local mean-field Hamiltonians $\left(H_{A}\right.$ and $\left.H_{B}\right)$ in a $16 \times 16$ matrix form for an $S=3 / 2$ dimer and calculate the expectation values of the ordered moments in the mean-field ground state. We solve this mean-field problem iteratively until the expectation values converge at both dimer sites. We retain all 16 eigenstates of the mean-field solution to describe the magnetic excitations.
TABLE III. Mode energies, intensity ratios, and normalized ordered moments of $\mathrm{Cr}_{2} \mathrm{TeO}_{6}$ obtained by INS measurements and ESW calculations.

\begin{tabular}{lcccc}
\hline \hline $\mathrm{Cr}_{2} \mathrm{TeO}_{6}$ & $E_{T}(\mathrm{meV})$ & $E_{L}(\mathrm{meV})$ & $I_{L} / I_{T}$ & $M / M_{s}$ \\
\hline Expt. & $15.4(4)$ & $23.7(8)$ & 0.08 & 0.76 \\
ESW & $15.8(1)$ & $23.0(1)$ & 0.29 & 0.82 \\
\hline \hline
\end{tabular}

For weak interdimer interactions, we obtain a singlet $\left(S_{\mathrm{tot}}=0\right)$ local ground state with no magnetic moment. When interdimer interactions increase, magnetic states of a dimer $\left(S_{\text {tot }} \neq 0\right)$ participate in the ground state and finite magnetic moments appear. The ordered phase is realized for

$$
20\left(\left|J_{2}\right|+\left|J_{3}\right|\right) \geqslant J_{1},
$$

and a QCP is obtained when

$$
20\left(\left|J_{2}\right|+\left|J_{3}\right|\right)=J_{1} .
$$

The finite Néel temperatures of $\mathrm{Cr}_{2} \mathrm{WO}_{6}$ and $\mathrm{Cr}_{2} \mathrm{TeO}_{6}$ indicate that the exchange interactions of these two compounds satisfy Eq. (6). On the basis of a dimer lattice, the ordered moment is staggered in $\mathrm{Cr}_{2} \mathrm{WO}_{6}$, therefore the ordering wave vector is $\mathbf{Q}=(001)$, whereas in $\mathrm{Cr}_{2} \mathrm{TeO}_{6}$, the ordered moment is uniform and the ordering wave vector is $\mathbf{Q}=(000)$.

We now discuss the magnetic excitations calculated by the extended spin-wave (ESW) theory for interacting spin dimer systems. The detailed ESW theory calculations can be found in the SM [39]. Figures 2(e) and 2(f) show the calculated powder-averaged magnetic excitation spectra based on the ESW theory for $\mathrm{Cr}_{2} \mathrm{WO}_{6}$ and $\mathrm{Cr}_{2} \mathrm{TeO}_{6}$, respectively, which are in very good agreement with the experimental data presented in Figs. 2(a) and 2(b). The exchange parameters used to obtain the best fit between measured and calculated spectra are tabulated in Table I, together with the calculated ordered moments (see Ref. [39] for how the exchange parameters are determined). A more quantitative comparison between the ESW theory calculated results and INS data has been given in Tables II and III, showing that the energy of the spectral weight maximum, the bandwidth of $T$ modes, the energy of high-energy gapped modes, and the ordered moment relative to the full moment of $\mathrm{Cr}^{3+}$ [38] can be reasonably well reproduced. Based on the ESW theory, the low-energy excitations in both compounds are indeed transverse Goldstone modes which can be also reproduced by the LSW theory as discussed previously. The additional gapped modes that cannot be captured by the LSW theory with or without anisotropy are better described by the ESW theory compared with other scenarios such as two-magnon excitations (Sec. VI [39]).

In the framework of the ESW theory, the excitation modes at $E \approx 12 \mathrm{meV}$ in $\mathrm{Cr}_{2} \mathrm{WO}_{6}$ and $E \approx 23 \mathrm{meV}$ in $\mathrm{Cr}_{2} \mathrm{TeO}_{6}$

TABLE II. Mode energies, intensity ratios, and normalized ordered moments of $\mathrm{Cr}_{2} \mathrm{WO}_{6}$ obtained by INS measurements and ESW calculations.

\begin{tabular}{lccccr}
\hline \hline $\mathrm{Cr}_{2} \mathrm{WO}_{6}$ & $E_{T}(\mathrm{meV})$ & $E_{L}(\mathrm{meV})$ & $E_{S_{\mathrm{tot}}=2}(\mathrm{meV})$ & $I_{L} / I_{T}$ & $I_{S_{\mathrm{tot}}=2} / I_{T}$ \\
\hline Expt. & $8.9(4)$ & $12.0(4)$ & $20.6(4)$ & 0.20 & 0.021 \\
ESW & $9.1(1)$ & $11.9(1)$ & $21.2(1)$ & 0.34 & 0.063 \\
\hline \hline
\end{tabular}



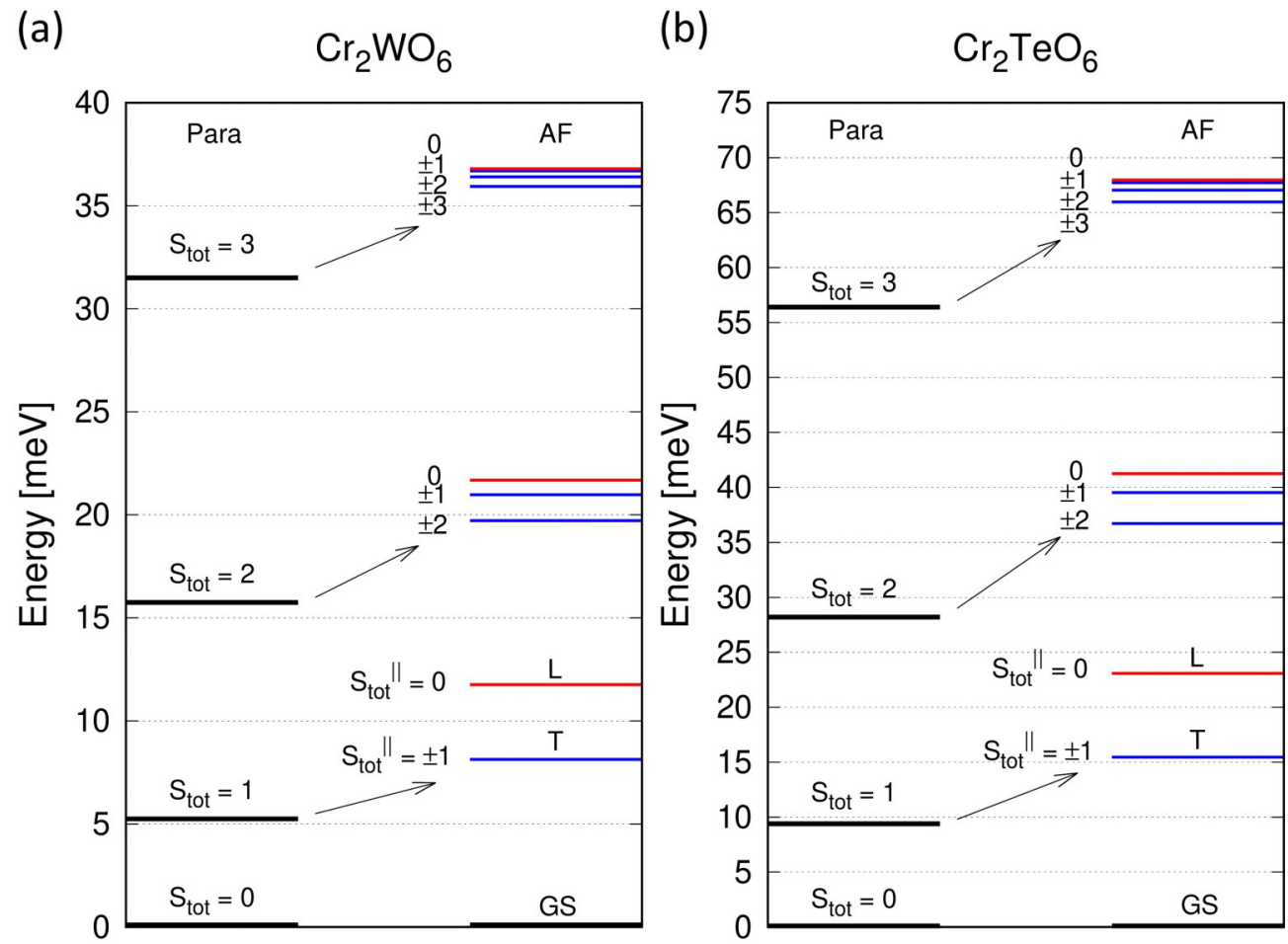

FIG. 7. Local energy levels of an $S=3 / 2$ dimer in paramagnetic and AF ordered phases for $\mathrm{Cr}_{2} \mathrm{WO}_{6}$ and $\mathrm{Cr}_{2} \mathrm{TeO}_{6}$. $S_{\text {tot }} \mathrm{represents}_{\mathrm{p}}$ the value of the total spin and classifies the energy eigenstates in the paramagnetic phase. In the ordered phase, the AF moment appears and the degenerate levels split. GS represents the ground state, whereas $L$ (red line) and $T$ (blue line) represent the $L$ and $T$ modes, respectively. The $L$ mode is singlet, while the $T$ mode is doubly degenerate. $S_{\text {tot }}^{\|}$means the component of the total spin parallel to the moment. The energy eigenstates are classified by $S_{\text {tot }}^{\|}$in the ordered phase. The numbers $\pm 3, \pm 2, \pm 1$, and 0 represent the values of $S_{\text {tot }}^{\|}$for the corresponding energy levels.

with a narrow bandwidth are interpreted as amplitude modes, which stem from fluctuations in the magnitude of the sublattice magnetic moment in the ordered state (with the ground state being a mixture of both the $S_{\text {tot }}=0$ spin-singlet state and $S_{\text {tot }}=1$ spin-triplet state). Note that the intensity of longitudinal modes relative to transverse modes is weaker in the experimental data compared to the calculated value. The overestimated intensity of the longitudinal modes might be due to the fact that the ESW theory calculations are done on the basis of a harmonic theory where the magnon-magnon interaction is not taken into account. In reality, the presence of this interaction would lead to a decay of the longitudinal mode into a pair of transverse modes, resulting in a broadened linewidth and reduced intensity.

An important prediction of the ESW theory is that there are additional higher-energy gapped modes around 21 and $40 \mathrm{meV}$ in $\mathrm{Cr}_{2} \mathrm{WO}_{6}$ and $\mathrm{Cr}_{2} \mathrm{TeO}_{6}$, respectively [Figs. 2(e) and 2(f)]. These are attributed to the $S_{\text {tot }}=2$ excited state stemming from the $S_{\text {tot }}=2$ local energy state of an $S=3 / 2$ spin dimer. In the presence of a staggered molecular field in the ordered state, the degeneracy of the $S_{\text {tot }}=2$ local dimer states is lifted, giving rise to local $L$ and $T$ states (Fig. 7), accompanied by longitudinal and transverse fluctuations of the ordered moment, respectively. The interdimer interactions enable these local excited states to move and dispersive excitation modes are formed. For $\mathrm{Cr}_{2} \mathrm{WO}_{6}$, the $S_{\text {tot }}=2$ energy level splits into two $T$ states and an $L$ state around $21 \mathrm{meV}$, leading to almost flat excitation modes as the dispersion is weak. This is in contrast to the $T$ mode around $9 \mathrm{meV}$ originating from the $S_{\text {tot }}=1$ state which has a stronger dispersion. Although the INS intensity of the $S_{\text {tot }}=2$ mode is weak, this mode has been convincingly observed in $\mathrm{Cr}_{2} \mathrm{WO}_{6}$, as shown in Figs. 3(b) and 4(a). This observation cannot be explained within the LSW theory, thus strongly favoring the ESW theory to describe the magnetic excitations and the interpretation of the 12- and 23-meV modes in $\mathrm{Cr}_{2} \mathrm{WO}_{6}$ and $\mathrm{Cr}_{2} \mathrm{TeO}_{6}$, respectively, as amplitude modes. We have tried to look for the $S_{\text {tot }}=2$ excitation modes in $\mathrm{Cr}_{2} \mathrm{TeO}_{6}$ using an incident neutron energy $E_{i}=90 \mathrm{meV}$, but the phonons around $40 \mathrm{meV}$ prevent the observation of this weak magnetic signal (Fig. S4 [39]).

It is known that in quantum antiferromagnets longitudinal excitations may emerge together with transverse modes in the same energy range when the ground state of a system is in the vicinity of a QCP where the ordered moment is suppressed significantly by quantum fluctuations. The observation of amplitude modes in the magnetic excitation spectra of $\mathrm{Cr}_{2} \mathrm{WO}_{6}$ and $\mathrm{Cr}_{2} \mathrm{TeO}_{6}$ even in the polycrystalline form is striking, considering that their ordered moments are reduced by only $\sim 24 \%$. A natural question is, are the ground states of these two compounds close to a QCP? To quantitatively evaluate the exchange interactions of $\mathrm{Cr}_{2} \mathrm{WO}_{6}$ and $\mathrm{Cr}_{2} \mathrm{TeO}_{6}$ with respect to a QCP, in Figs. 8(a) and 8(b) we show $\left|J_{2}\right| / J_{1}$ dependence of the excitation gap and sublattice magnetic moment with fixed $J_{1}$ and $J_{3}$ listed in Table I. In the disordered phase, the excitation gap is analytically given by 
(a)

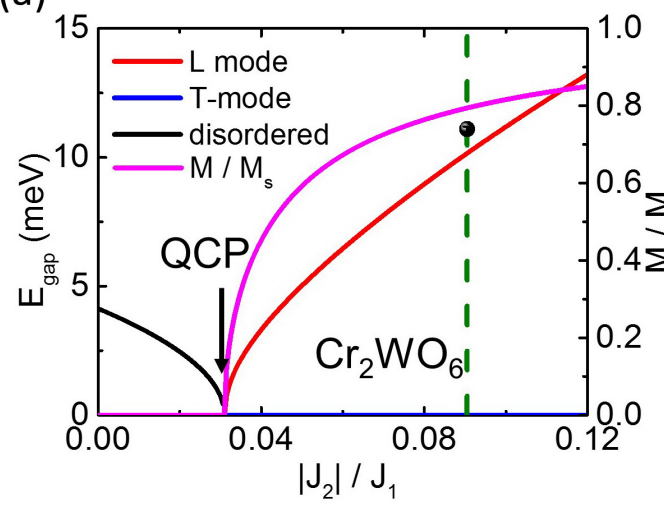

(b)

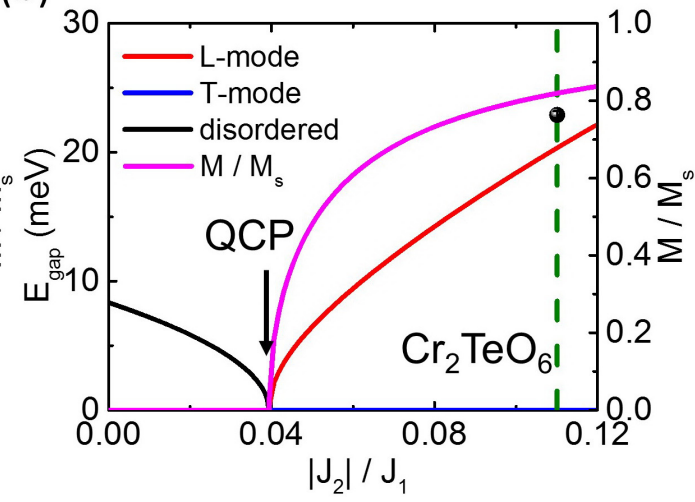

FIG. 8. (a), (b) $\left|J_{2}\right| / J_{1}$ dependence of the normalized ordered moment $M / M_{s}$ and the excitation gaps, where all the 16 local states of an $S=3 / 2$ spin dimer have been taken into account. The black line represents the excitation gap in the disordered state. The excitation gap of the longitudinal modes ( $L$ mode) and the transverse mode ( $T$ mode) in the ordered state are plotted by red and blue solid lines, respectively. The $\left|J_{2}\right| / J_{1}$ ratios of $\mathrm{Cr}_{2} \mathrm{TeO}_{6}$ and $\mathrm{Cr}_{2} \mathrm{WO}_{6}$ estimated from the INS data are denoted by vertical green lines. The calculated ordered moments are represented by magenta lines. The black spheres denote the measured sublattice magnetic moments from Ref. [38] where the error bars are smaller than the symbols.

$E_{\text {gap }}=\sqrt{J_{1}^{2}-20 J_{1}\left(\left|J_{2}\right|+\left|J_{3}\right|\right)}$, which is threefold degenerate and decreases with increasing $\left|J_{2}\right| / J_{1}$ as represented by the black lines. In the ordered phase, the excitation modes split into two $T$ modes and one $L$ mode. The $T$ modes stay gapless, reflecting the global rotational symmetry of the spin Hamiltonian. The gap of the $L$ mode scales with $\left|J_{2}\right| / J_{1}$, corresponding to the Higgs mass in high-energy physics. In the meantime, the sublattice magnetic moment increases with $\left|J_{2}\right| / J_{1}$, as illustrated by the magenta curves. According to Eq. (7), the QCP is located at $\left|J_{2}\right| / J_{1}=0.031$ for $\mathrm{Cr}_{2} \mathrm{WO}_{6}$ and 0.040 for $\mathrm{Cr}_{2} \mathrm{TeO}_{6}$. The ratios of $\mathrm{Cr}_{2} \mathrm{WO}_{6}$ and $\mathrm{Cr}_{2} \mathrm{TeO}_{6}$ estimated by the INS data have been indicated by the green dashed lines in Fig. 8, which are $\left|J_{2}\right| / J_{1}=0.0905$ and 0.101 , respectively. Correspondingly, the calculated ordered moments normalized to the fully saturated value of $\mathrm{Cr}^{3+}$ agree reasonably well with the observed reduction of $\sim 24 \%$ (black sphere).

As a system goes away from the QCP, the energy of the amplitude modes increases and the INS intensity weakens. This, together with the tendency of decaying into transverse modes, makes amplitude modes difficult to be detected experimentally. Our observation brings about another question: What makes it feasible to observe the longitudinal modes in these two compounds? There are several contributing factors: (i) These compounds are dimers with spin $S=3 / 2$. Similar to $S=1 / 2$ spin dimers, the low-lying states here are $S_{\text {tot }}=0$ singlet and $S_{\text {tot }}=1$ triplet. However, the matrix elements of spin operators (between the singlet and triplet states) are different between $S=3 / 2$ and $S=1 / 2$ dimer systems, with the matrix element enhanced by $\sqrt{ } 5$ for the $S=3 / 2$ dimer (Sec. VII [39]). As a result, the intensity of the amplitude modes is enhanced relative to that of the transverse modes in the case of $S=3 / 2$. (ii) The dispersion of the amplitude modes in both $\mathrm{Cr}_{2} \mathrm{WO}_{6}$ and $\mathrm{Cr}_{2} \mathrm{TeO}_{6}$ is weak and the INS intensity stays in a narrow energy range separated from the transverse mode (Figs. S8 and S9 [39]), which makes it possible to distinguish these modes even with polycrystalline samples by gaining INS intensity in a narrow energy region.

\section{CONCLUSION}

In this paper, we have studied magnetic excitations of $S=3 / 2$ spin dimers $\mathrm{Cr}_{2} \mathrm{WO}_{6}$ and $\mathrm{Cr}_{2} \mathrm{TeO}_{6}$ by inelastic neutron scattering. In addition to the transverse modes corresponding to phase fluctuations of the order parameter, we have observed additional gapped modes arising from amplitude fluctuations of the sublattice magnetization. These findings are better described by an extended spin-wave theory compared to the linear spin-wave theory. The large ordered moment relative to the fully saturated moment of $\mathrm{Cr}^{3+}$ indicates that these two compounds are not close to a quantum critical point. Therefore, our results suggest that amplitude modes are not the privilege of ordered quantum spin dimers in the vicinity of a QCP, but may be common excitation modes that can survive even away from the QCP. Our study paves an avenue to search for and understand the physics of amplitude modes in other interacting spin dimer systems beyond $S=1 / 2$.

\section{ACKNOWLEDGMENTS}

Work at Michigan State University was supported by the National Science Foundation under Award No. DMR1608752 and the startup funds from Michigan State University. A portion of this research used resources at the Spallation Neutron Source, a DOE Office of Science User Facility operated by the Oak Ridge National Laboratory. M.M. is supported by JSPS KAKENHI Grant No. 17K05516. Work at University of Tennessee was supported by the National Science Foundation under Award No. DMR-1350002.
[1] S. R. Elliott and M. Franz, Rev. Mod. Phys. 87, 137 (2015).
[2] D. Pekker and C. M. Varma, Annu. Rev. Condens. Matter Phys. 6, 269 (2015). 
[3] P. W. Anderson, Phys. Rev. 110, 827 (1958).

[4] P. W. Higgs, Phys. Rev. Lett. 13, 508 (1964).

[5] P. W. Higgs, Phys. Lett. 12, 132 (1964).

[6] R. Sooryakumar and M. V. Klein, Phys. Rev. Lett. 45, 660 (1980).

[7] P. B. Littlewood and C. M. Varma, Phys. Rev. B 26, 4883 (1982).

[8] R. Matsunaga, Y. I. Hamada, K. Makise, Y. Uzawa, H. Terai, Z. Wang, and R. Shimano, Phys. Rev. Lett. 111, 057002 (2013).

[9] R. Matsunaga, N. Tsuji, H. Fujita, A. Sugioka, K. Makise, Y. Uzawa, H. Terai, Z. Wang, H. Aoki, and R. Shimano, Science 345, 1145 (2014).

[10] M. Endres, T. Fukuhara, D. Pekker, M. Cheneau, P. Schauß, C. Gross, E. Demler, S. Kuhr, and I. Bloch, Nature (London) 487 , 454 (2012).

[11] R. S. Fishman and S. H. Liu, Phys. Rev. B 54, 7233 (1996).

[12] R. S. Fishman and S. H. Liu, Phys. Rev. B 54, 7252 (1996).

[13] G. Grüner, Density Waves in Solids (CRC Press, Boca Raton, FL, 2000).

[14] B. Lake, D. A. Tennant, and S. E. Nagler, Phys. Rev. Lett. 85, 832 (2000).

[15] Ch. Rüegg, B. Normand, M. Matsumoto, A. Furrer, D. F. McMorrow, K. W. Krämer, H.-U. Güdel, S. N. Gvasaliya, H. Mutka, and M. Boehm, Phys. Rev. Lett. 100, 205701 (2008).

[16] H. Kuroe, N. Takami, N. Niwa, T. Sekine, M. Matsumoto, F. Yamada, H. Tanaka, and K. Takemura, J. Phys.: Conf. Ser. 400, 032042 (2012).

[17] K. Penc, J. Romhányi, T. Rõõm, U. Nagel, Á. Antal, T. Fehér, A. Jánossy, H. Engelkamp, H. Murakawa, Y. Tokura, D. Szaller, S. Bordács, and I. Kézsmárki, Phys. Rev. Lett. 108, 257203 (2012).

[18] P. Merchant, B. Normand, K. W. Krämer, M. Boehm, D. F. McMorrow, and Ch. Rüegg, Nat. Phys. 10, 373 (2014).

[19] B. Grenier, S. Petit, V. Simonet, E. Canévet, L.-P. Regnault, S. Raymond, B. Canals, C. Berthier, and P. Lejay, Phys. Rev. Lett. 114, 017201 (2015).

[20] T. Hong, M. Matsumoto, Y. Qiu, W. Chen, T. R. Gentile, S. Watson, F. F. Awwadi, M. M. Turnbull, S. E. Dissanayake, H. Agrawal, R. Toft-Petersen, B. Klemke, K. Coester, K. P. Schmidt, and D. A. Tennant, Nat. Phys. 13, 638 (2017).

[21] T. Hong, Y. Qiu, M. Matsumoto, D. A. Tennant, K. Coester, K. P. Schmidt, F. F. Awwadi, M. M. Turnbull, H. Agrawal, and A. L. Chernyshev, Nat. Commun. 8, 15148 (2017).
[22] R. L. Dally, Y. Zhao, Zhijun Xun, R. Chisnell, M. B. Stone, J. W. Lynn, L. Balents, and S. D. Wilson, Nat. Commun. 9 , 2188 (2018).

[23] I. Affleck and G. F. Wellman, Phys. Rev. B 46, 8934 (1992).

[24] A. V. Chubukov and D. K. Morr, Phys. Rev. B 52, 3521 (1995).

[25] M. Troyer, M. E. Zhitomirsky, and K. Ueda, Phys. Rev. B 55, R6117 (1997).

[26] B. Normand and T. M. Rice, Phys. Rev. B 56, 8760 (1997).

[27] M. Matsumoto, B. Normand, T. M. Rice, and M. Sigrist, Phys. Rev. B 69, 054423 (2004).

[28] T. Kato, A. Oosawa, K. Takatsu, H. Tanaka, W. Shiramura, K. Nakajima, and K. Kakurai, J. Phys. Chem. Solids 60, 1125 (1999).

[29] Z. Tun, W. J. L. Buyers, R. L. Armstrong, K. Hirakawa, and B. Briat, Phys. Rev. B 42, 4677 (1990).

[30] M. Enderle, Z. Tun, W. J. L. Buyers, and M. Steiner, Phys. Rev. B 59, 4235 (1999).

[31] A. V. Chubukov, S. Sachdev, and J. Ye, Phys. Rev. B 49, 11919 (1994).

[32] S. Sachdev, Phys. Rev. B 59, 14054 (1999).

[33] D. Podolsky, A. Auerbach, and D. P. Arovas, Phys. Rev. B 84, 174522 (2011).

[34] A. Jain, M. Krautloher, J. Porras, G. H. Ryu, D. P. Chen, D. L. Abernathy, J. T. Park, A. Ivanov, J. Chaloupka, G. Khaliullin, B. Keimer, and B. J. Kim, Nat. Phys. 13, 633 (2017).

[35] R. Shiina, H. Shiba, P. Thalmeier, A. Takahashi, and O. Sakai, J. Phys. Soc. Jpn. 72, 1216 (2003).

[36] M. Drillon, L. Padel, and J. C. Bernier, Physica B+C 97, 380 (1979).

[37] W. Kunnmann, S. La Placa, L. M. Corliss, J. M. Hastings, and E. Banks, J. Phys. Chem. Solids 29, 1359 (1968).

[38] M. Zhu, D. Do, C. R. Dela Cruz, Z. Dun, H. D. Zhou, S. D. Mahanti, and X. Ke, Phys. Rev. Lett. 113, 076406 (2014).

[39] See Supplemental Material at http://link.aps.org/supplemental/ 10.1103/PhysRevResearch.1.033111 for extended inelastic neutron scattering data, details on the linear spin-wave and extended spin-wave theory calculations, and discussions on two-magnon contributions and effects of matrix elements on the relative $L$-mode intensity.

[40] T. Sommer, M. Vojta, and K. W. Becker, Eur. Phys. J. B 23, 329 (2001).

[41] M. Matsumoto, B. Normand, T. M. Rice, and M. Sigrist, Phys. Rev. Lett. 89, 077203 (2002). 